\title{
O ENSINO DE NANOCIÊNCIAS POR MEIO DE OBJETOS DE APRENDIZAGEM
}

Anderson Luis Ellwanger - Centro Universitário Franciscano, pfandd@ gmail.com

Jussane Rossato - Centro Universitário Franciscano, jussaner@gmail.com

Mateus Granada - Centro Universitário Franciscano, magranada@gmail.com

Valéria I. Bortoluzzi - Centro Universitário Franciscano, valeria.bortoluzzi@gmail.com Solange Binotto Fagan - Centro Universitário Franciscano, solange.fagan@gmail.com

\section{Resumo}

Neste trabalho, apresentamos resultados de transposições didáticas relacionadas com tópicos de Nanociências, desenvolvidos por alunos do Mestrado Profissionalizante em Ensino de Física e Matemática, alocado no Centro Universitário Fraciscano (UNIFRA). Os produtos educacionais foram avaliados por docentes da área e seguiram a proposta metodológica do projeto MAIS Unifra, que vislumbra a inter-relação entre áreas do conhecimento e a não linearidade de sua construção. Os conteúdos abordados estão relacionados com conceitos básicos usados em Nanociências e propriedades ópticas em nanoescala. Os produtos educacionais foram organizados de forma a contemplarem demonstrações computacionais, animações, vídeos e hipertextos. Ao final dos conteúdos há atividades que podem ser desenvolvidas pelos usuários e também sugestões para a sua aplicação em sala de aula presencial ou a distância. Atualmente, a equipe continua trabalhando na construção de unidades didáticas e na produção de objetos pedagógicos relacionados a conteúdos associados às Nanociências.

Palavras-chave: transposição didática; multidisciplinar; conteúdos digitais.

\begin{abstract}
This work presents the evaluation of didactic transpositions of Nanoscience topics, developed by students of the Professional Master in Teaching of Physics and Mathematics, allocated in the University Center Franciscano (UNIFRA). The educational products were judged by teachers of the specific area and use of the methodology proposed in MAIS Unifra project, which aims the interrelations between knowledge areas and the non-linearity in this construction. The subjects covered are related to Nanoscience basic concepts, specially optical properties at the nanoscale. The educational product were organized in order to present computer demonstrations, animations, video and hypertext. Additionally, it is also present on the educational products some activities that can be developed by users and also suggestions for its implementations in the classroom or from a distance. Currently, the team continues to work on construction of teaching units and productions of objects related to pedagogical content related to Nanoscience.
\end{abstract}

Keywords: didactic transpositions; multidisciplinary, digital subject.

\section{INTRODUÇÃO}

As Nanociências e a Nanotecnologia usam conceitos tanto da Física Clássica, como da Física Quântica, para auxiliar na compreensão do comportamento de átomos e 
moléculas que passam a ser manipulados para formar novos materiais, muitas vezes mais resistentes e duráveis. Compostos esses que apresentam propriedades diferentes de quando trabalhados em escala macroscópica. No momento em que os átomos e moléculas são manipulados, podemos reduzir os desperdícios na sua produção, excluindo impurezas e imperfeições (ROUKES, 2008).

A Nanotecnologia tem sido considerada uma área promissora para produção de novos dispositivos tecnológicos que poderão mudar o modo como vivemos (TOMA, 2004). Dessa forma, torna-se essencial a compreensão desta tecnologia, de seus princípios básicos. Uma abordagem de assuntos mais amplos possibilita a interação de várias disciplinas e gera uma compreensão mais profunda do assunto estudado, muitas vezes transcendendo o limite da Física e adentrando em outras áreas do conhecimento, como Biologia e Química, fazendo das Nanociências uma área de natureza multidisciplinar (TOMA, 2004).

Nesta perspectiva, e considerando que novos produtos e aplicações vêm sendo apresentadas a cada dia por especialistas da área, faz-se necessário o entendimento, pelo público em geral, dos conceitos envolvidos na construção desses materiais na escala nano. Porém, sabe-se que a compreensão dos processos e conceitos envolvidos em dispositivos em escala nanométrica é compartilhada por poucos, visto que em muitos casos são conhecimentos abstratos e são necessárias ferramentas matemáticas sofisticadas, impedindo ao público leigo participar das discussões e relativizar possíveis implicações.

Com o objetivo de popularizar o conhecimento científico, pela transposição didática de tópicos de Nanociências numa linguagem acessível, de forma multidisciplinar e não linear, capaz de ser compreendida pela população leiga no assunto, apresenta-se essa proposta diferenciada, que tem como finalidade levar a esse público informações de forma objetiva e simples sobre conceitos envolvidos em Nanociências.

Neste trabalho, faremos a descrição dos objetos de aprendizagem presentes nos conteúdos didáticos "Conceitos básicos relacionados com as Nanociências" e "Propriedades Óticas em Nanociências". Ao final das atividades desenvolvidas pelos usuários os mesmos devem ser capazes de compreender o aumento da área superficial; entender como os conceitos de reflexão, refração e dispersão estão relacionados com materiais em escalas nanométricas; comparar como o magnetismo ocorre em escala macroscópica e nanométrica; compreender o efeito da mudança de cor em partículas de diferentes tamanhos e entender como os conceitos de espectro e radiação eletromagnética estão relacionados com a Nanociência. Sendo assim, discutiremos a relevância de tais tópicos do ponto de vista educacional, que será abordada na próxima seção.

\section{NANOCIÊNCIAS NO ENSINO BÁSICO}

A Nanociência tem recebido grande destaque em veículos midiáticos devido às aplicações que a mesma pode trazer para a sociedade. Dispositivos sensíveis ao toque, maleáveis e resistentes, películas transparentes com propriedades antibactericidas tecidos hidrofóbicos, cosméticos que apresentam maior absorção pela pele, entre outras aplicações, tornam a manipulação atômica muito atrativa (GOODSELL, 2004).

Muitos destes recursos nanotecnológicos são usados pela população, melhorando a forma de viver e deixando a vida mais prática. Contudo, poucos conseguem compreender como tais dispositivos nesta escala tão pequena são capazes de funcionarem tão bem. 
A compreensão desses conceitos que estão relacionados com as novas tecnologias perpassa pela compreensão do que é Nanociência, sendo esta definida como a possibilidade de manipular objetos em escala nanométrica, mais precisamente, trabalha-se com frações correspondentes à bilionésima parte do metro.

No entanto, acreditamos que uma das propostas para que a população comece a familiarizar-se com esses conceitos, nesta escala, inicie pelo ensino fundamental e médio. Sabendo-se que a Nanociência é uma área multidisciplinar, esses conceitos poderão ser abordados em diferentes disciplinas, como Biologia, Química, Física, entre outras. A inserção dessa área nos currículos das escolas segue as orientações sugeridas pelos Parâmetros Curriculares Nacionais (BRASIL, 2002), o qual sugere que o desenvolvimento cognitivo do aluno deve ser diretamente ligado a assuntos relacionados ao seu cotidiano, para que possam ter uma visão geral e crítica do mundo onde vivem.

Há necessidade de alfabetizar cientificamente nossos estudantes, para que estes compreendam o mundo tecnológico que os cerca, possibilitando desta forma que entendam o funcionamento de alguns equipamentos que fazem parte do seu dia a dia. Porém, muitas vezes os alunos não possuem informações suficientes para tal entendimento (HEALY, 2009), o que pode ser atenuado com a organização de materiais didáticos atualizados, inclusive entre eles os que se referem às Nanociências.

As perspectivas para o futuro, nesta área, são as mais diversas, como a possibilidade de aumento da memória e velocidade dos computadores, cabos feitos de nanotubos e nanofios de carbono, novos materiais inteligentes, construção de nanomáquinas, as quais poderão inserir medicamentos no corpo humano e em locais específicos (BARONE, 2005). Então, precisamos de conteúdos relacionados às Nanociências que sejam diferenciados e de fácil entendimento aos usuários, que expliquem minimamente estas novas aplicações.

Com este trabalho buscamos disponibilizar ao público interessado conteúdos didáticos relacionados com os temas Nanociências e Nanotecnologia, estimulando a sociedade em geral no entendimento sobre estes temas tão divulgado na mídia.

Assim, buscamos, por meio da elaboração de conteúdos didáticos informativos, contendo conceitos de Nanociências, aproximar os usuários desse ramo da ciência, bem como da própria escala de tamanho, já que a percepção da Nanociência está muitas vezes associada à imagem de uma atividade desenvolvida em laboratórios sofisticados e caros e isto pode criar barreiras por parte do público na curiosidade por investigar o tema. Esses conteúdos didáticos também poderão servir de apoio aos professores para que os mesmos possam utilizá-los na abordagem do tema em sala de aula, de forma que os alunos possam observar como e onde se relacionam as Nanociências e suas aplicações.

Por último podemos observar que a tecnologia e a ciência atual caminham definitivamente para a escala nanométrica, tanto através da miniaturização na eletrônica, como através da montagem nanoestrutural a partir de átomos e moléculas. Nesse sentido, a Nanotecnologia e a Nanociências não podem deixar de ser incluídas no cenário da educação, em seus diferentes níveis, visto que os futuros professores e cientistas são os jovens estudantes, e necessitam desenvolver uma visão crítica das inovações tecnológicas desenvolvidas no mundo.

Na próxima seção, enfatizaremos como a transposição didática pode tornar-se mais eficiente quando usada a metodologia do MAIS Unifra (MAIS UNIFRA, 2012). 


\section{TRANSPOSIÇÃO DIDÁTICA}

A transposição didática entre a produção científica e o contexto educacional é almejada por praticamente todos os componentes curriculares. Essa baseia-se na possibilidade de levar o conhecimento abstrato, definido como "saber sábio", gerado pelos cientistas, para as salas de aula, o "saber ensinado" (CHEVALLARD, 1991). Porém, na maioria das propostas encontradas na literatura especializada, estas tentativas alcançam resultados pouco expressivos, versando basicamente sobre a possibilidade de apresentar aos alunos tópicos motivacionais acerca das inovações científicas (PEREIRA; HONÓRIO; SANNOMIYA, 2010).

As Nanociências enquadravam-se neste contexto, porém, esforços significativos por parte de pesquisadores e estudantes do Centro Universitário Franciscano UNIFRA-RS - vêm possibilitando a transposição didática mais eficiente, onde os tópicos mais pertinentes, detectados em periódicos da área, são explorados, usando exemplos de aplicação e conhecimentos prévios de múltiplas áreas, permitindo vincular o ensino regular com tópicos relacionados a esta ciência promissora.

A transposição didática é feita por uma equipe pedagógica, constituída por duas professoras doutoras em Física da Matéria Condensada com trabalhos na área de Nanociências e um professor mestre e doutorando em Ensino de Física com pesquisa na área de Ensino de Nanociências, que adequam a linguagem que expressa conceitos e noções sobre o tema. A equipe técnica é responsável por transformar o que foi adapatado para roteiros em conteúdos digitais e objetos de aprendizagem. Esse trabalho é pensado para a educação básica e, em alguns casos, também para a educação superior. Alguns dos trabalhos desenvolvidos por discentes do mestrado profissional em Ensino de Física e Matemática auxiliam nesta transposição.

As atividades propostas contemplam conteúdos que podem ser mediados por professores, e também materiais autoinstrutivos, disponibilizados num espaço virtual designado como MAIS Unifra (MAIS UNIFRA, 2012), que permite acesso a usuários de diferentes níveis de desenvolvimento cognitivo, possibilitando que estes explorem os conteúdos digitais e instruam-se de maneira gradual e individual, o que vem a se adequar às orientações metodológicas e epistemológicas dos PCNs (BRASIL, 2002).

As aplicações em ambientes formais de ensino apresentaram resultados encorajadores, os quais foram apontados por depoimentos de alunos que participaram das atividades desenvolvidas durante a pesquisa de Ellwanger (2010) e de Granada (2011).

Os bons índices de aceitação da proposta incentivaram a equipe de pesquisadores a investir na divulgação dos materiais, a acrescer novos tópicos, a ampliar os conceitos abordados e a aprofundar as discussões geradas por estes, possibilitando alcançar um número ainda mais expressivo de pessoas, popularizando dessa forma a Nanociência.

Na próxima seção, discutiremos como o uso de novas tecnologias é importante para facilitar o processo de ensino-aprendizagem.

\section{NOVAS TECNOLOGIAS NO ENSINO}

Os computadores estão presentes em todas as áreas do conhecimento segundo Araujo e Veit (2004), inclusive no ensino e na pesquisa que contemplam as Nanociências. Na área de ensino, enfocada neste trabalho, as demonstrações computacionais facilitam a visualização de estruturas e efeitos nanométricos. No caso de simulações computacionais que são usadas em pesquisas de ponta, essas auxiliam na 
avaliação das propriedades estruturais e eletrônicas de nanoestruturas e suas possíveis aplicações, as quais estão sendo apresentadas via transposição didática para os usuários.

O uso de computadores, se for amparado metodologicamente por teorias de aprendizagem, como as de Ausubel (2002) - Novak (1981) e Gowin(1981), e ainda nos modelos mentais de Johnson - Laird (1987), de modo a contribuir para a visualização durante a instrução, podem ser grandes facilitadores do processo de ensino e aprendizagem.

As formas de uso mais comuns de computadores, segundo Araujo e Veit (2004) enquadram-se nos seguintes grupos: a) instrução e avaliação mediada, onde é possível diagnosticar concepções prévias dos alunos; b) modelagem e simulação, as quais permitem a interação com o modelo existente; c) coleta e análise de dados, possibilitando que o usuário se preocupe mais com a compreensão dos conceitos envolvidos do que com a obtenção de dados do experimento; d) recurso multimídia, por meio de hipertextos e links faz com que o usuário busque o caminho que julgar mais adequado para sua aprendizagem; e) comunicação a distância, permitindo a discussão em blogs e chats dos tópicos referentes ao conteúdo trabalhado; f) resolução algébrica e demonstrações, nestes são usados textos de divulgação científica e as deduções matemáticas são detalhadas; g) processos cognitivos, a máquina fornece o contexto pedagógico e permite o estudo de processos cognitivos do usuário.

Cabe salientar que durante o uso de recursos informatizados, os limites destes devem ser discutidos, permitindo que estes sejam compreendidos como uma ferramenta auxiliar e não um processo único. Então, pelo exposto, pode-se concluir que o uso de dispositivos informatizados pode ser útil, desde que usado como dispositivo complementar, auxiliando na compreensão dos assuntos abordados, contribuindo assim para aumentar as possibilidades de uma aprendizagem menos mecânica e mais significativa, quiçá tornando o ensino de Ciências mais atrativo.

$\mathrm{Na}$ próxima seção, descreveremos a metodologia utilizada para o desenvolvimento dos conteúdos didáticos e dos objetos de aprendizagem.

\section{METODOLOGIA DE TRABALHO DO "MAIS UNIFRA"}

O Centro Universitário Franciscano (UNIFRA) conta, atualmente, com um um espaço virtual em permanente transformação, fazendo uso da metodologia MAIS Unifra (MAIS UNIFRA, 2012). Neste espaço há conteúdos digitais e objetos de aprendizagem de autoria da equipe de professores e alunos de graduação e pósgraduação da instituição. Também conta-se com o auxílio de profissionais da equipe técnica que são responsáveis por transformar os roteiros de conteúdos digitais em produtos tecnológicos.

A interatividade se faz presente por meio de hipertextos e links direcionais. Já o diferencial desta proposta está nos fóruns moderados e chats interativos, onde os usuários podem interagir com outros usuários e encaminhar sugestões sobre os conteúdos e o ambiente à equipe responsável por estes. Sendo assim, o MAIS Unifra (MAIS UNIFRA, 2012) caracteriza-se por um ambiente de aprendizagem o qual se concretiza de forma compartilhada e em rede.

Dentre os conteúdos desenvolvidos pelas equipes pedagógicas e técnicas, daremos ênfase neste trabalho aos conteúdos didáticos "Conceitos básicos relacionados com Nanociências" e "Propriedades Óticas em Nanociências". Estes temas foram abordados com o objetivo de introduzir tópicos indispensáveis para a compreensão mínima de conceitos envolvidos em Nanociências. 
Nas próximas subseções, faremos a descrição dos objetos de aprendizagem presentes nos dois conteúdos didáticos, "Conceitos básicos relacionados com Nanociências" e "Propriedades Óticas em Nanociências" pertencentes à unidade didática Nanociências, disponíveis no espaço virtual de aprendizagem MAIS Unifra (MAIS UNIFRA, 2012).

\subsection{Conceitos básicos relacionados com Nanociências}

Os objetivos de aprendizagem deste conteúdo foram: compreender o aumento da área superficial; entender como os conceitos de reflexão, refração e dispersão estão relacionados com materiais em escalas nanométricas; comparar como o magnetismo ocorre em escala macroscópica e nanométrica. Para alcançar estes objetivos, foram organizadas algumas atividades que iniciam com uma apresentação que objetiva a motivação do usuário.

Para que o primeiro objetivo fosse contemplado, foi organizado um conteúdo explicativo bem como um objeto de aprendizagem. Este objeto interativo consiste na visualização do aumento da área superficial, no qual um cubo é dividido em 8 ou 64 vezes. Para que o conceito de área superficial seja entendido é calculado o volume e a área superficial do cubo para as duas possibilidades (quando o cubo é dividido em $8 \mathrm{e}$ 64 vezes), concluindo-se, ao final do objeto que quanto mais fragmentado estiver o cubo maior será a área superficial do mesmo.

Na Figura 01 temos a representação esquemática do objeto "área superficial".

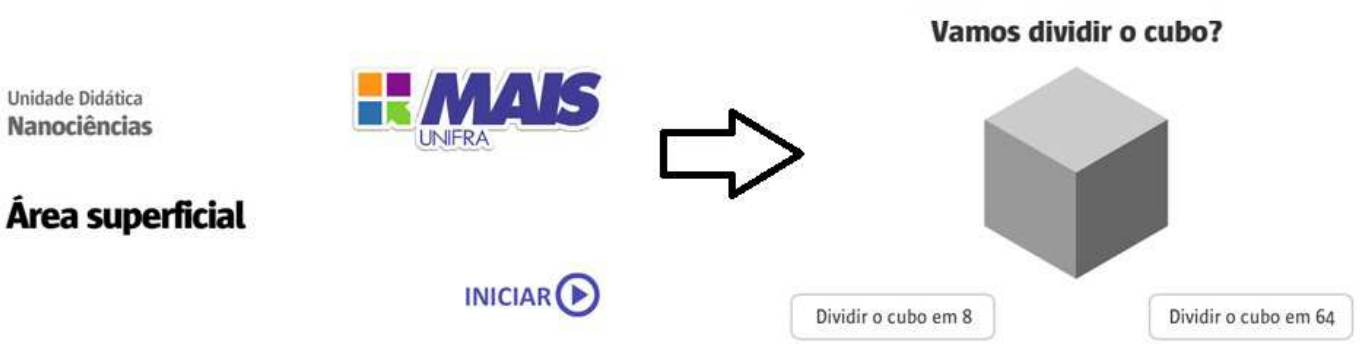

Figura 01 - Representação esquemática da página inicial do objeto de aprendizagem área superficial num cubo (MAIS UNIFRA, 2012).

Para alcançar o segundo objetivo, foi organizado um texto usando como motivação a pluridade de cores presentes nos vitrais das igrejas medievais, arguindo acerca dos efeitos ópticos envolvidos nesse processo. Para que o usuário possa ter entendimento acerca dos efeitos ópticos, foi desenvolvido o objeto "mudança de cor do ouro", onde se pode escolher o tamanho nanométrico das partículas de ouro, sendo que, na sequência, o simulador moerá esta partícula que, em contato com um solvente como a água, irá explicitar cores diferenciadas que estão associadas ao tamanho da partícula escolhida. Se o usuário almejar explicações mais detalhadas sobre o motivo da mudança de cor, basta clicar no ícone "Por que muda de cor?". Ao final deste objeto, o usuário irá concluir que as propriedades de alguns elementos químicos, como o ouro, apresentam diferentes características quando manipulados em escalas menores e uma das mudanças está na cor que o elemento pode apresentar. ouro".

Na Figura 02, temos a representação esquemática do objeto "mudança de cor do 


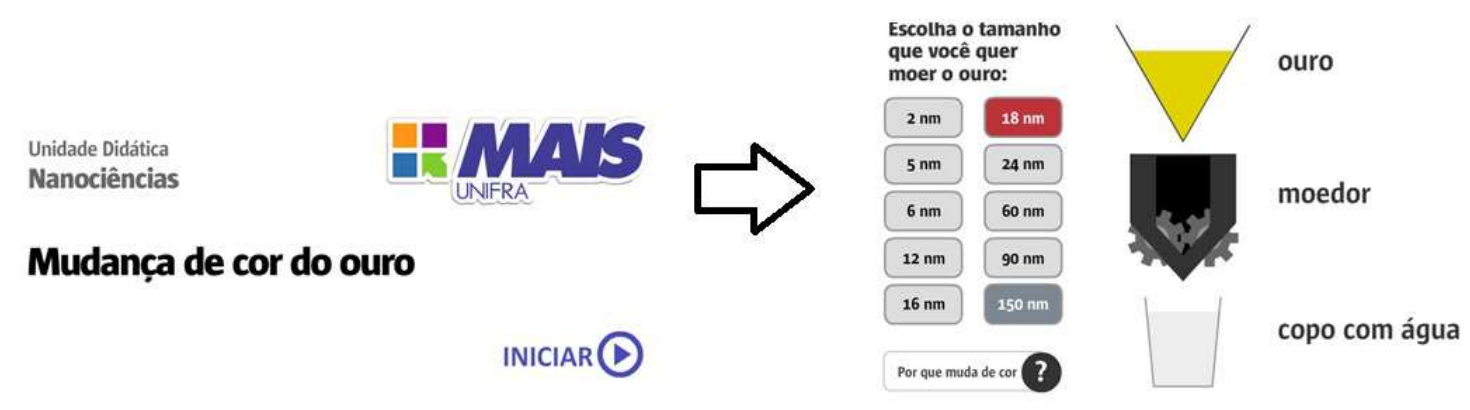

Figura 02 - Representação esquemática da página inicial do objeto de aprendizagem mudança de cor do ouro (MAIS UNIFRA, 2012).

Na próxima subseção serão discutidos os objetos de aprendizagem presentes no conteúdo Propriedades Ópticas em Nanociências.

\subsection{Propriedades Ópticas em Nanociências}

Este conteúdo didático tem como objetivos de aprendizagem: compreender o efeito da mudança de cor em partículas de diferentes tamanhos e entender como os conceitos de espectro e radiação eletromagnética estão relacionados com as Nanociências.

Para alcançar os objetivos propostos foram organizados alguns objetos de aprendizagem neste conteúdo didático. Para que o primeiro objetivo fosse contemplado, o usuário poderá buscar informações no objeto de aprendizagem "mudança de cor do ouro" proposto no conteúdo digital "Conceitos Básicos relacionados com Nanociências" (MAIS UNIFRA, 2012). Além desse objeto, ainda foi organizado um objeto denominado "confinamento quântico" para ilustrar que a grande maioria das alterações nas propriedades das substâncias em nanoescala está diretamente associada ao confinamento quântico eletrônico. Além disso, a alteração na radiação emitida pode causar mudança de cor, onde os espectros de energia contínuos passam a ser discretos.

$\mathrm{Na}$ Figura 03, temos a representação esquemática do objeto "confinamento quântico" .

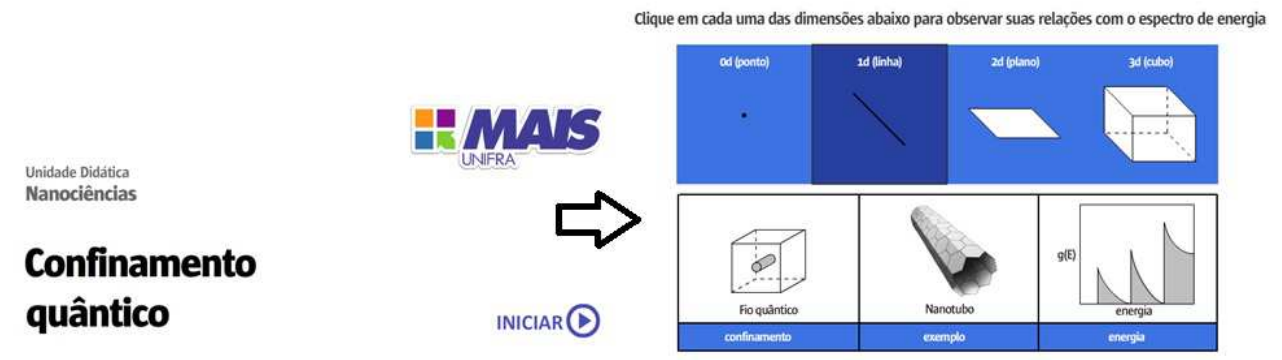

Figura 03 - Representação esquemática da página inicial do objeto de aprendizagem confinamento quântico (MAIS UNIFRA, 2012).

Para interagir com este objeto de aprendizagem o usuário poderá escolher uma das dimensões $(0 \mathrm{D}, 1 \mathrm{D}, 2 \mathrm{D}, 3 \mathrm{D})$ para observar suas relações com o espectro de energia, 
bem como, exemplos e tipo de confinamento quântico. Para maiores informação o usuário poderá obter explicações no conteúdo "Nanomateriais de Carbono".

O segundo objetivo foi alcançado por meio de um texto discursivo e também por meio do objeto de aprendizagem "espectro eletromagnético". Neste objeto o usuário poderá escolher a faixa de frequência do espectro, sendo possível obter informações sobre as aplicações e equipamentos que usam aquela determinada frequência, bem como possíveis implicações destas.

$\mathrm{Na}$ Figura 04 temos a representação esquemática do objeto de aprendizagem "espectro eletromagnético".

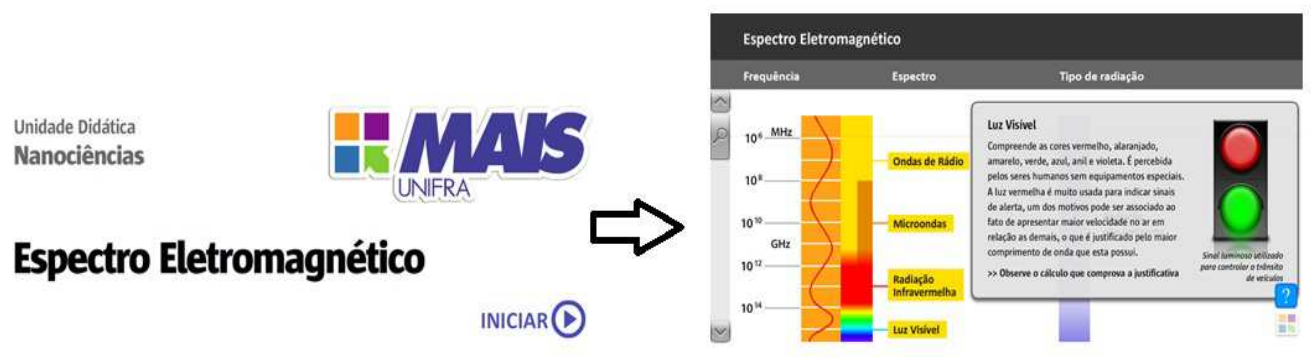

Figura 04 - Representação esquemática da página inicial do objeto de aprendizagem - espectro eletromagnético (MAIS UNIFRA, 2012).

Acreditamos que estes conteúdos e os objetos de aprendizagem desenvolvidos na unidade didática Nanociências possam servir de apoio ao público (professores, alunos etc.) que tem interesse por este tema tão divulgado na mídia, pemitindo assim o acesso ao conhecimento científico atual.

\section{CONCLUSÕES}

Considerando que uma grande parcela da população domina as tecnologias atuais e possui interesse por estas, foi usado este fator motivador para inserir alguns tópicos contemporâneos de forma diferenciada em ambientes virtuais de aprendizagem. Este processo pode permitir a complementação das atividades desenvolvidas em sala de aula pelo professor, tornando, desta forma, o ensino mais atrativo e interligado com o cotidiano dos alunos. Também é possível que o usuário navegue pelos conteúdos didáticos e instrua-se sem a presença "concreta" do professor, onde as dúvidas podem ser esclarecidas por meio de chats e fóruns de discussões, os quais tornam-se uma poderosa ferramenta de troca de conhecimentos entre os usuários.

A mídia vem divulgando novas descobertas cientificas, entre elas estão as Nanociências. Porém o grande público não tem conhecimentos prévios para compreender os conceitos envolvidos nesta área. Desta forma a transposição didática usada para adequar esses conceitos a uma linguagem mais acessível era necessária, permitindo que o conhecimento científico fosse acessível a uma grande quantidade de pessoas.

Neste trabalho, fizemos a transposição didática, por meio de objetos de aprendizagem, inseridos em hipertextos buscando facilitar a compreenssão dos conceitos abordados em Nanociências. Para facilitar a assimilação destes conceitos foram construídos alguns objetos de aprendizagem que estão inserido nos conteúdos 
didáticos "Conceitos básicos relacionados com Nanociências" e "Propriedades Óticas em Nanociências". Nesses objetos, a visualização do aumento da área superficial, a mudança de cor do ouro, o confinamento quântico e o espectro eletromagnético foram contemplados, atingindo assim o objetivo de facilitar o entendimento de conceitos abstratos de forma simples.

Assim, a produção de objetos de aprendizagem que contemplem tecnologias atuais aliadas a novas metodologias de ensino, poderão auxiliar professores e alunos de diferentes níveis de ensino em suas práticas educacionais, tornando o ensino mais atualizado e atrativo. Esta metodologia busca uma maior interatividade entre os usuários e também um incentivo à busca por conhecimentos acerca de temas atuais.

\section{AGRADECIMENTOS}

UNIFRA e Instituto Nacional de Nanomateriais de Carbono - INCT/ Nanocarbono.

\section{REFERÊNCIAS BIBLIOGRÁFICAS}

ARAUJO, I. S.; VEIT, E. A. Uma Revisão da Literatura sobre Estudos Relativos a Tecnologias Computacionais no Ensino de Física. Investigações em Ensino de Ciências (UFRGS), São Paulo, v. 4, n. 3, p. 5-18, 2004.

AUSUBEL, D. P. Retenção e aquisição de conhecimento: uma perspectiva cognitiva. Lisboa: Plátano, 2002.

BARONE, P. W. et al. Near-infrared optical sensors based on single-walled carbon nanotubes. Nature Materials, v. 4, p. 86-92, 2005.

BRASIL. Ministério da Educação, Secretaria de Educação Média e Tecnológica. Parâmetros curriculares nacionais: Ensino Médio. Brasília, MEC/SEMT, 2002.

CHEVALLARD, Y. La Transposición Didáctica: del saber sabio al saber enseñado. Aique:Argentina, 1991.

EllWANGer, A. L. Tópicos de Nanociências em Conteúdos de Física no Ensino Básico. Santa Maria, RS, 2010, 70 p. Dissertação (Mestrado Profissionalizante em Ensino de Física e de Matemática)- Centro Universitário Franciscano.

GOODSELL, D. S. Biotechnology: lessons from nature. Wiley-Liss:New Jersey, 2004.

GRANADA, M. Propriedades ópticas de Materiais no Ensino Médio por meio da Nanociências. Santa Maria, RS, 2011, 93 p. Dissertação (Mestrado Profissionalizante em Ensino de Física e de Matemática)- Centro Universitário Franciscano.

GOWIN, D. B. Educating. Ithaca: NY, Cornell University Press (1981).

HEALY, N. Why Nano Education? Journal of Nano Education, v.1, p. 6-7, 2009.

JOHNSON-LAIR, P. N. Modelos mentales en ciencia cognitiva. In: Norman, Donald, A. Pespectivas de la ciencia cognitiva. Barcelona:, Paidós, 1987. 
MAIS UNIFRA. Espaço Virtual de Aprendizagem. Santa Maria, RS: Unifra, 2011. Disponível em: <http://maisunifra.com.br/>. Acesso em: 10 mai. 2012.

NOVAK, J. D. Uma teoria de educação. São Paulo: Pioneira, 1981. Tradução de M. A. Moreira do original A Theory of education, Cornell University Press, 1977.

PEREIRA, F.; HONÓRIO, K; SANNOMIYA, M. Nanotecnologia: Desenvolvimento de materiais didáticos para uma abordagem no ensino Fundamental. Química Nova na Escola, v. 32, n. 2, 2010.

ROUKES, M. Espaço suficiente lá embaixo. Scientific American Brasil. Edição Especial: Nanotecnologia, 2008.

TOMA, E. H. O Mundo Nanométrico: a dimensão do novo século. Oficina de Textos: São Paulo, 2004. 